Revue des patrimoines et plasticiens (XXe-XXle siècles)

\title{
Guillaume Gillet, « la liaison de l'art monumental et des arts plastiques ", principe ou réalité ? Le cas des vitraux de l'église Notre-Dame à Royan
}

The association between monumental art and plastic arts, principle or reality?

Guillaume Gillet and the stained-glass windows at the Notre-Dame de Royan church

\section{Franck Delorme}

\section{OpenEdition}

Journals

Édition électronique

URL : http://journals.openedition.org/insitu/14998

DOI : $10.4000 /$ insitu. 14998

ISSN : 1630-7305

Éditeur

Ministère de la Culture

Référence électronique

Franck Delorme, « Guillaume Gillet, « la liaison de l'art monumental et des arts plastiques », principe ou réalité ? Le cas des vitraux de l'église Notre-Dame à Royan », In Situ [En ligne], 32 | 2017, mis en ligne le 05 septembre 2017, consulté le 21 décembre 2020. URL : http://journals.openedition.org/insitu/14998 ; DOI : https://doi.org/10.4000/insitu.14998

Ce document a été généré automatiquement le 21 décembre 2020.

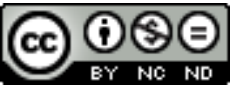

In Situ Revues des patrimoines est mis à disposition selon les termes de la licence Creative Commons Attribution - Pas d'Utilisation Commerciale - Pas de Modification 4.0 International. 


\section{Guillaume Gillet, « la liaison de l'art monumental et des arts}

\section{plastiques », principe ou réalité ? Le cas des vitraux de l'église Notre- Dame à Royan}

The association between monumental art and plastic arts, principle or reality? Guillaume Gillet and the stained-glass windows at the Notre-Dame de Royan church

\section{Franck Delorme}

Communication présentée lors de la journée d'étude « Le collectif sur la place publique » organisée à l'Institut national d'histoire de l'art le 26 mars 2014 par l'université Paris-1-Panthéon-Sorbonne et l'université Lille-3.

Située en Charente-Maritime, sur l'estuaire de la Gironde, Royan, qui fut en très grande partie détruite par des bombardements en 1944, est dominée par la haute silhouette de l'église Notre-Dame, devenue en 1958 le symbole de la renaissance de la ville entière (fig. 1). 
Figure 1

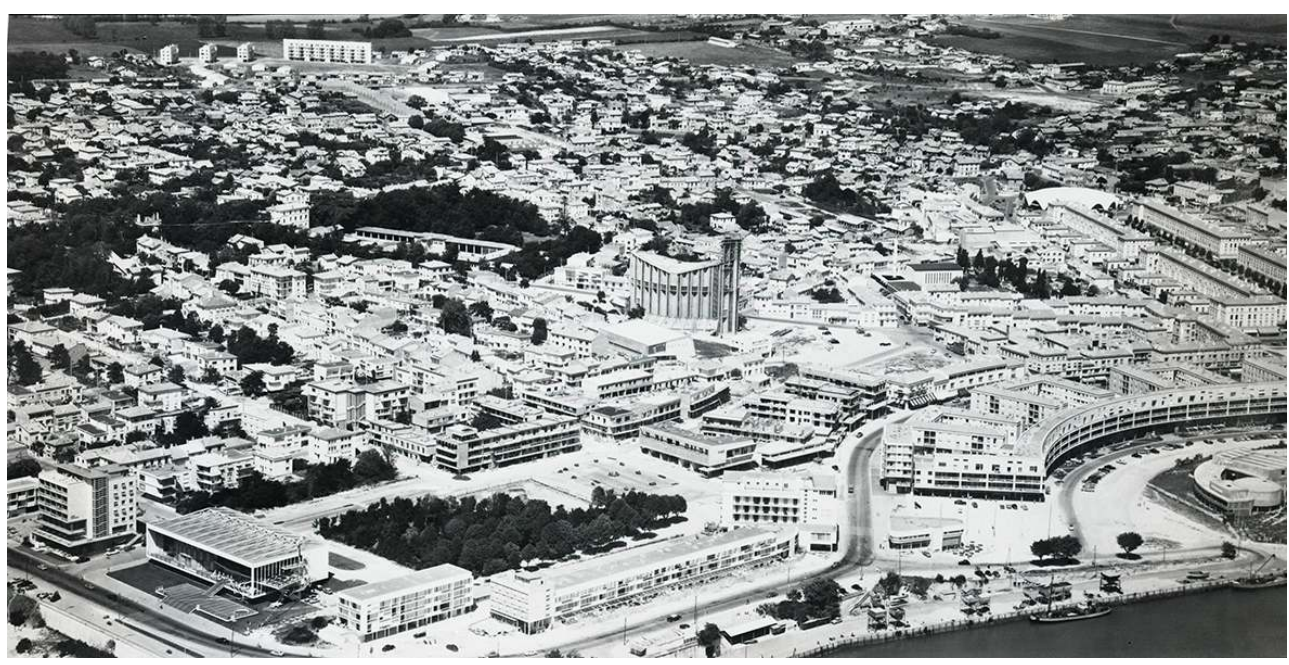

Vue aérienne générale du centre de Royan reconstruit avec l'église Notre-Dame en cours d'achèvement, vers 1958. SIAF/CAPA, fonds Gillet, 152 IFA 1504/1.

(C) Cité de l'architecture et du patrimoine, Paris.

2 L'église se dresse au sein de «la ville la plus cinquante de France» dont le plan de reconstruction et d'urbanisme fut conçu par les architectes Claude Ferret (1907-1993) et Louis Simon (1901-1965) à partir de 1945¹. Grâce à son architecte, Guillaume Gillet, l'église de Royan a pris place dans l'histoire de l'architecture religieuse ${ }^{2}$ et elle s'inscrit dans le mouvement de renouveau de l'art sacré qui se produisit pendant les Trente Glorieuses, période d'innovation technique autant qu'esthétique ${ }^{3}$. Pour sa conception et son édification, Guillaume Gillet a fait appel à la science des ingénieurs, à des techniques nouvelles et à des matériaux contemporains. Il a su donner un souffle et une atmosphère qui font écho, à plusieurs siècles de distance, à l'esthétique et à l'esprit de l'architecture gothique, écrivant ainsi une sorte de langage gothique moderne ${ }^{4}$ (fig. 2). 


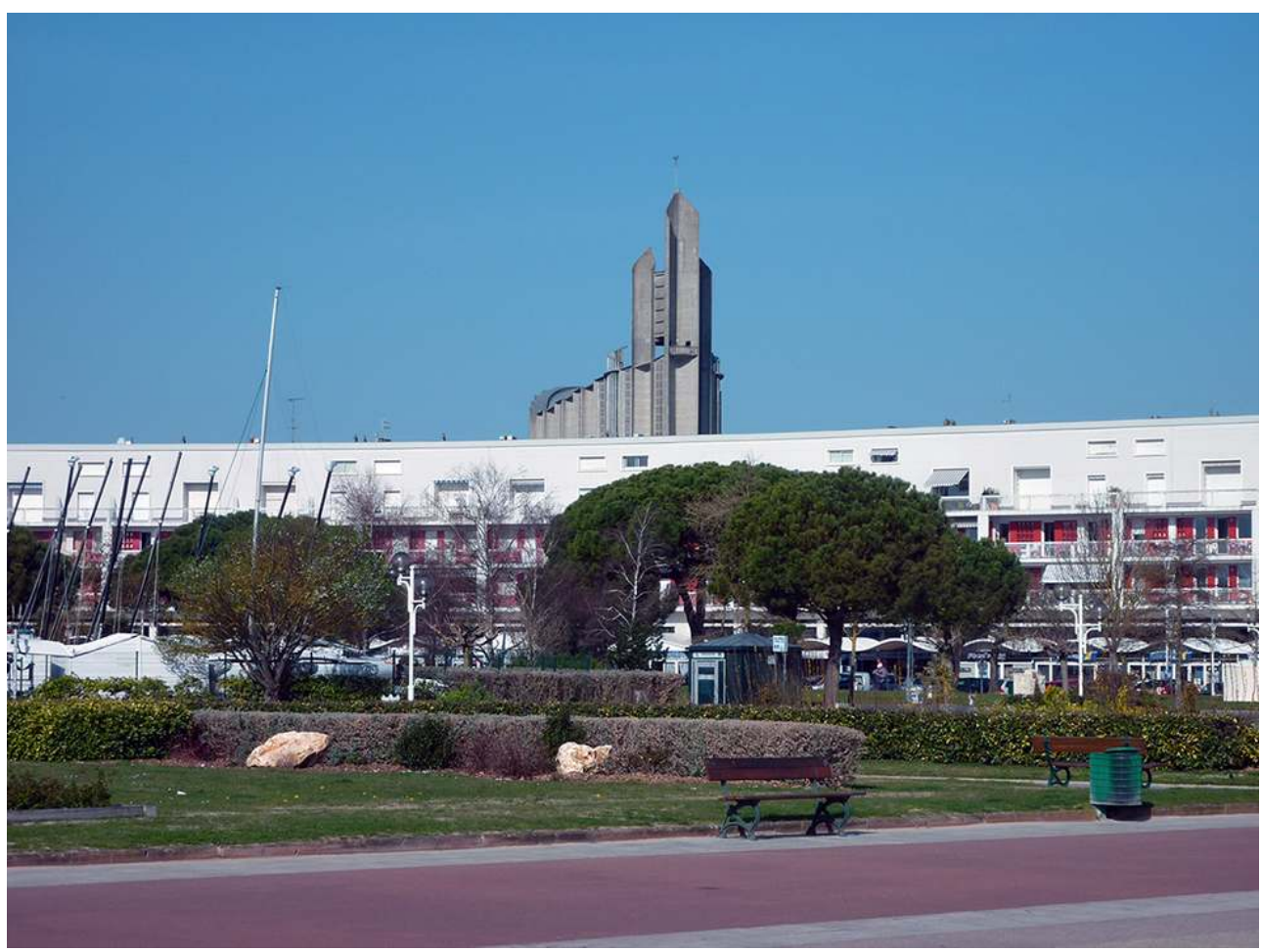

La silhouette de l'église Notre-Dame de Royan dominant les immeubles du front de mer reconstruit par Louis Simon et Claude Ferret.

Phot. Franck Delorme, 2012. ( ) Franck Delorme.

La grande nef de l'église de Royan, constituée par un faisceau d'éléments autoporteurs en béton armé, invention de l'ingénieur Bernard Laffaille ${ }^{5}$ (1900-1955), est éclairée par une série de baies hautes et étroites (fig. 3) que complète un ensemble de verrières en forme de losanges percées dans la couverture d'une galerie périphérique semblable à un bascôté. Toutes ces ouvertures sont closes par un ensemble de vitraux mis en œuvre selon la technique de la dalle de verre par le peintre-verrier Henri Martin-Granel (1914-2008) de 1958 à 1969, à l'exception du vitrail du chœur, œuvre de l'artiste Claude Idoux (1915-1990) qui ne fait pas partie du cycle mis en place sous la direction de Guillaume Gillet. La longue définition du programme et la lente installation des vitraux invitent à s'interroger sur les processus convoqués par l'architecte pour mener à bien l'œuvre jusque dans ses moindres détails. 


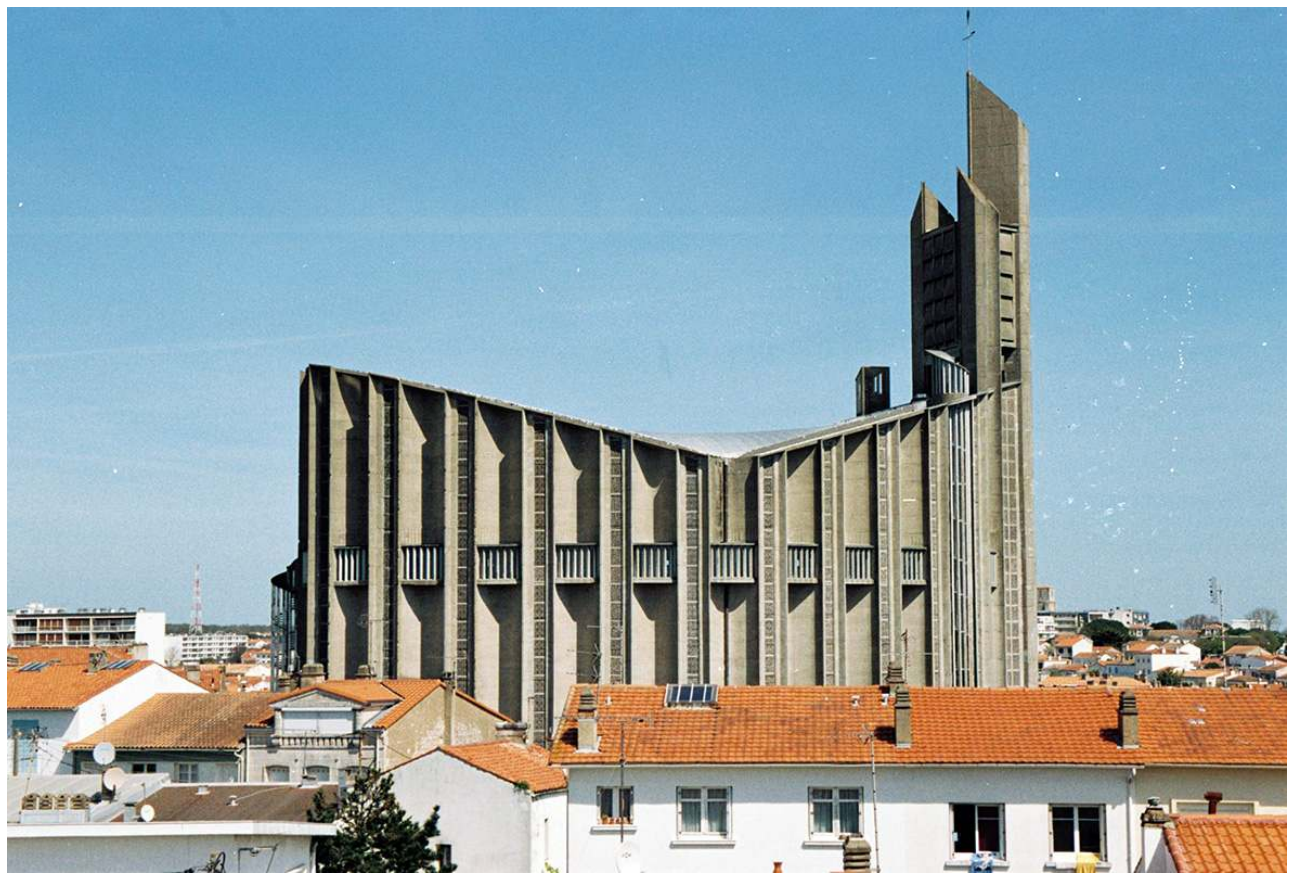

Le volume de la nef « gothique moderne » de l'église Notre-Dame de Royan émergeant au-dessus des toits de la ville.

Phot. Franck Delorme, 2012. (c) Franck Delorme.

\section{La liaison des arts selon Guillaume Gillet}

4 L'identification de l'auteur des vitraux de l'église Notre-Dame de Royan questionne encore aujourd'hui l'historien et le chercheur, malgré la considérable richesse des archives de l'architecte Guillaume Gillet ${ }^{6}$. Celles du peintre verrier Henri Martin-Granel ne sont pas plus éloquentes à ce sujet ${ }^{7}$. Aucune information, dans ces deux sources, ne permet d'attribuer de manière certaine la paternité de la conception des vitraux à l'un ou l'autre des deux protagonistes ni même de déterminer précisément la part de chacun dans une œuvre commune. Si les archives de Guillaume Gillet conservent des esquisses de vitraux (fig. 4) qui semblent montrer la voie vers ce qui sera réalisé, elles ne permettent pas de désigner de manière certaine l'architecte comme l'auteur des vitraux de l'église qu'il a conçue. 


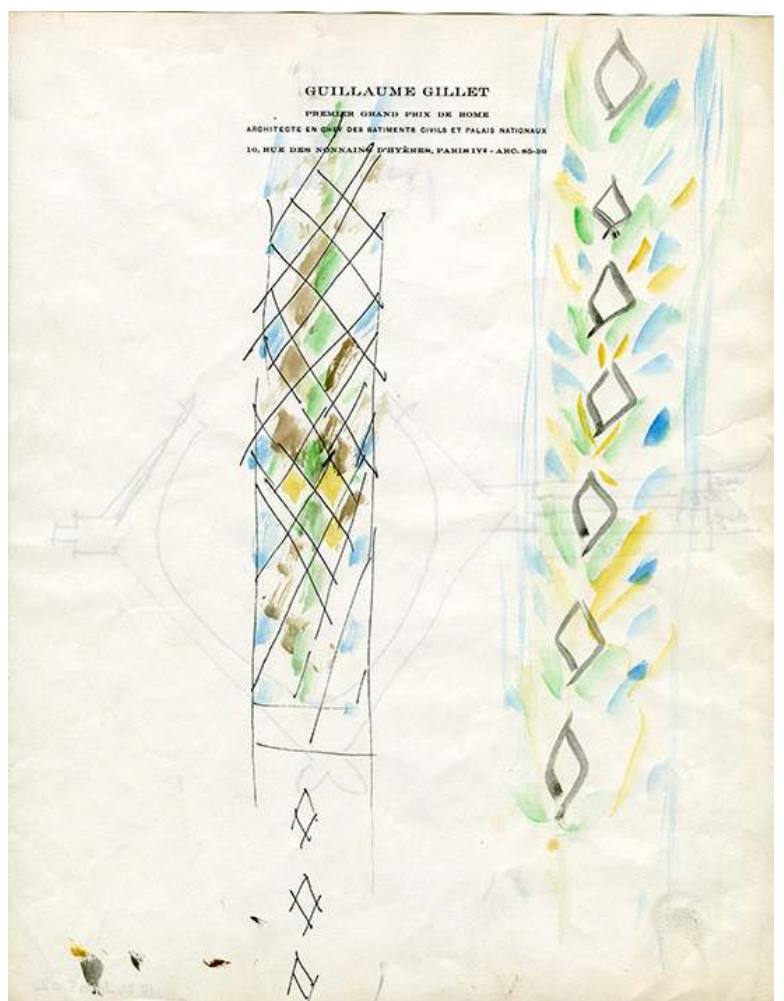

Esquisse de Guillaume Gillet pour les vitraux de l'église Notre-Dame de Royan, non datée. SIAF/CAPA, fonds Gillet, 152 IFA 2485/2.

(C) Cité de l'architecture et du patrimoine, Paris.

Les archives du peintre verrier contiennent des détails techniques de mise en œuvre et de fixation des verrières (fig. 5) mais aucun carton de vitraux. Ne pouvant avoir recours aux témoignages d'acteurs depuis trop longtemps disparus, et les archives ne conservant malheureusement pas de correspondance ni de comptes rendus d'échanges significatifs entre les acteurs à ce sujet, il faut se résoudre à naviguer de conjecture en conjecture et à émettre des hypothèses que nous nous efforcerons le plus possible de vérifier ou d'infirmer grâce aux rares documents existants. Plus largement, à partir de l'exemple de l'église de Royan, il est possible d'interroger la notion de liaison des arts telle que Guillaume Gillet l'a posée lui-même en 1965. Au moment où il se trouvait face à la difficulté de déterminer les principes d'exécution d'un programme de vitraux pour l'église Notre-Dame, Gillet intervenait en avril 1965 devant la commission de l'Équipement culturel du Ve Plan pour aborder « la liaison de l'Art monumental et des arts plastiques $»^{8}$. Dans cette communication, il faisait la constatation d'un divorce entre l'architecture - sous le terme d'art monumental - et les autres arts - peinture, sculpture, vitraux, céramique, tapisserie, etc. - dits « plastiques ». Par cette terminologie, il évacuait la notion péjorative de « décoratifs » qui renvoie immanquablement à une subordination, à un rang inférieur de certains arts par rapport à l'architecture, le dépassement d'une hiérarchie entre un art majeur, l'architecture, et des arts mineurs. Il n'en demeure pas moins que, devant ce divorce, Guillaume Gillet, dans son texte, appelait de ses vœux « une réconciliation [...] entre des conjoints qui ne demandent qu'à reprendre leur vie commune et à vivre sous le même toit ${ }^{9}$. La conception des vitraux de Royan fait parfaitement écho à cette «réconciliation", cette «vie commune » dont parle 
l'architecte. L'enjeu le plus important est en effet, plus qu'un dialogue, une interaction, une union de l'architecture et du vitrail, une fusion de l'espace et de la lumière : « un choix très délicat dont dépend tout le climat intérieur de l'édifice ${ }^{10}$.

Figure 5

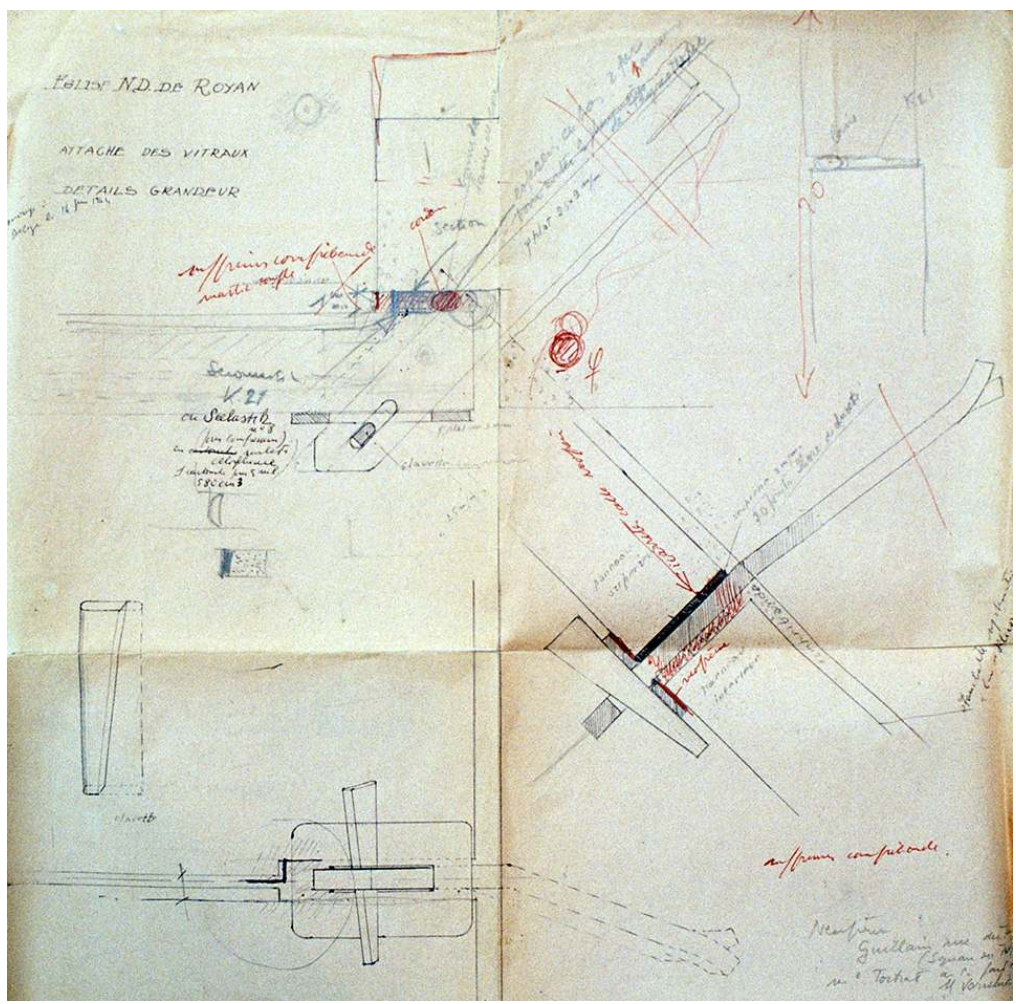

Détail grandeur des scellements des panneaux en dalle de verre de l'église Notre-Dame de Royan par Henri Martin-Granel, 1964. Archives privées de la famille Martin-Granel.

Phot. Franck Delorme. (c) Franck Delorme.

\section{Notre-Dame de Royan, un exemple de liaison des arts?}

Les quelques écrits de Guillaume Gillet consacrés soit aux vitraux de Notre-Dame de Royan - notamment un texte contenu dans ses mémoires, manuscrit resté inédit ${ }^{11}$-, soit, de manière plus large, à la question des relations entre art et architecture ou entre artistes et architectes, peuvent néanmoins servir de fil conducteur et constituer l'amorce d'un argumentaire. Arguments pour tenter d'appréhender la position ou les positions successives prises par l'architecte confronté au difficile choix d'un parti esthétique et d'un maître d'œuvre pour mener à bien l'ensemble d'un programme de vitraux pour une église qui demeure une icône du renouveau de l'architecture religieuse après la Seconde Guerre mondiale. Renouveau de l'architecture religieuse et non pas renaissance de l'art sacré car l'édifice ne saurait être confondu avec les expressions artistiques qu'il renferme, ou qu'il ne renferme pas. En effet, au contraire de Notre-Dame-de-Toute-Grâce (plateau d'Assy, Haute-Savoie) de Maurice Novarina (1907-2002), l'église de Royan n'est pas l'écrin d'une collection d'œuvres d'artistes renommés tels Georges Braque, Fernand Léger, Alfred Manessier, Georges Rouault... Assy, du point de vue architectural, reste en quelque 
sorte dans le droit fil de la tradition de l'architecture religieuse et constitue l'exact contraire de l'église de Royan. Pour Gérard Monnier, il s'agit d'« un bon exemple de l'influence des courants du régionalisme sur l'architecture religieuse ", ajoutant qu'à ce titre « elle a souvent été critiquée par les défenseurs de la modernité $»^{12}$.

7 Notre-Dame de Royan représente en effet une nouveauté par rapport à une église comme celle d'Assy. Par la radicalité de sa conception, l'édifice de Royan renouvelle les formes, le volume et même la spatialité de l'architecture religieuse. De plus, pour des raisons économiques mais également du fait d'une réelle conviction personnelle, son architecte renonce aux matériaux traditionnels et adopte le béton armé dans toute sa nudité et sa franchise. Loin d'être un colosse lourd et massif, l'église se révèle tout entière d'un élan ascensionnel encore plus marqué lorsqu'on pénètre à l'intérieur (fig. 6). Il semble bien qu'ici, l'architecte s'est souvenu des leçons du passé et en a tiré un enseignement qui ne s'incarne pas dans la forme mais bien dans l'esprit de la construction. Car c'est bien la construction et les choix constructifs qui, non seulement, déterminent la silhouette et l'espace, mais également "l'atmosphère", notion très importante en matière d'architecture religieuse et d'espace sacré.

Figure 6

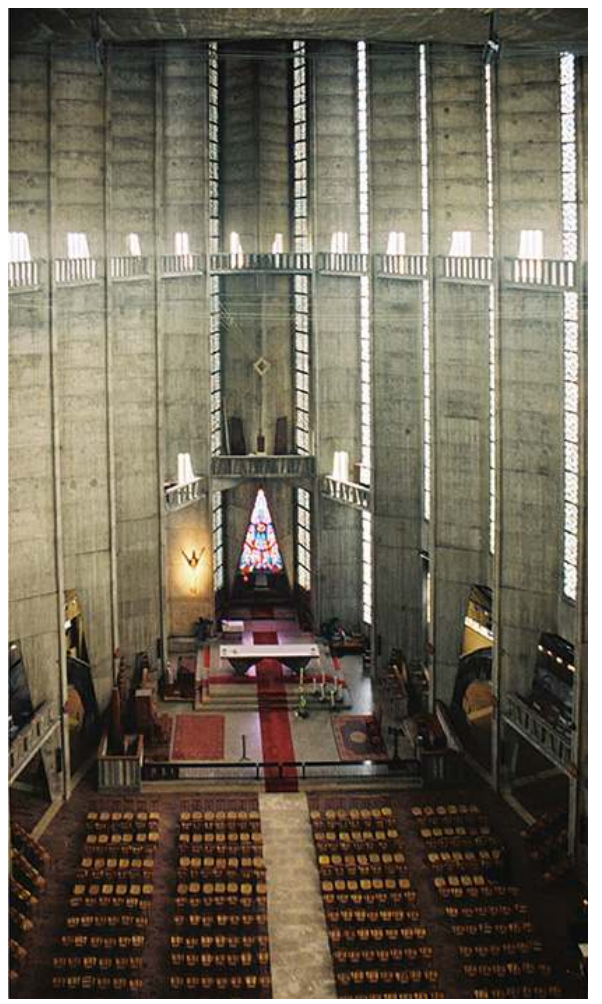

Vue de l'intérieur de la nef de l'église Notre-Dame de Royan.

Phot. Franck Delorme, 2012. (c) Franck Delorme.

Dans le texte inachevé des mémoires dont il a été fait mention plus haut, Guillaume Gillet n'aborde pas véritablement la question de l'intégration des arts dans son projet d'architecture pour Royan. Tout juste fait-il allusion au fait que pour lui, la richesse d'une église ne réside pas dans les trésors d'art qu'on y entasse (est-ce là une critique voilée de l'église de Novarina?) et qu'il convient de ne pas "confondre dépense d'argent et richesse d'esprit ${ }^{13}$ ». En revanche, il donne une première clé pour comprendre dans quel 
état d'esprit il pouvait être lors de la conception de son projet. Pour lui, « la pauvreté, la nudité des églises romanes n'est-elle pas la meilleure leçon de grandeur, et l'esprit de pauvreté de saint François d'Assise n'est-il pas la source de toute une ère nouvelle d'art, d'architecture et de prière? $»^{14}$. C'est la réponse qu'il opposait à la critique d'un évêque qui lui reprochait d'avoir élevé une église trop vaste et dénudée, d'y avoir consacré l'intégralité du financement et de n'avoir rien laissé pour orner l'édifice de statues, de tableaux, de vitraux, etc. Guillaume Gillet trouva son inspiration davantage dans les grands modèles d'architecture gothique que dans l'architecture romane. En effet, l'élévation de la nef de Notre-Dame de Royan évoque bien plus les grands vaisseaux des cathédrales gothiques dans lesquelles la formation et la définition de l'espace sacré découlent directement de la construction et de la structure (fig. 7). Dans une publication consacrée à l'église de Royan ${ }^{15}$, nous avancions le concept de " gothique moderne " pour qualifier cette « transposition » ou cette « réinterprétation » constructive et spatiale.

Figure 7

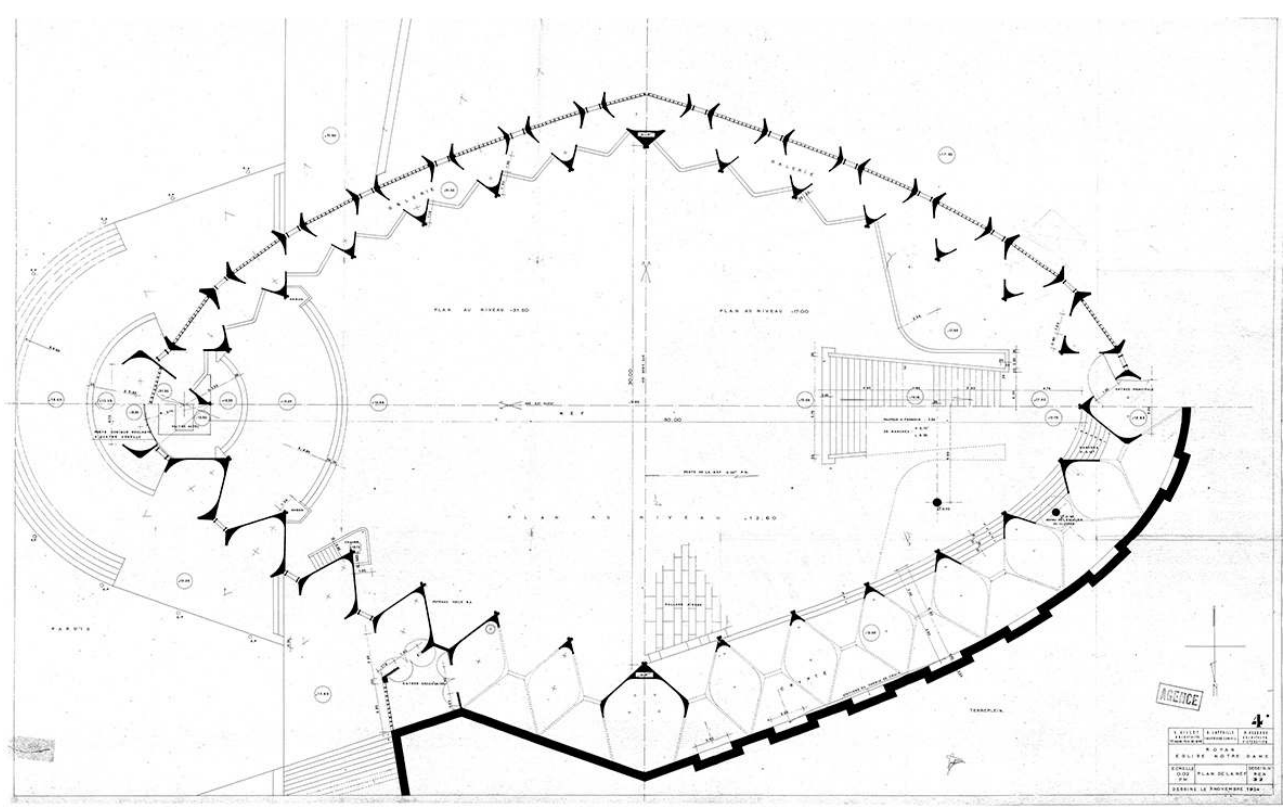

Plan au niveau de la nef et au niveau de la première galerie de l'église Notre-Dame de Royan, 3 novembre 1954. SIAF/CAPA, fonds Gillet, 152 IFA 2485/2.

(C) Cité de l'architecture et du patrimoine, Paris.

Royan est en effet caractérisée par la reprise, par Guillaume Gillet, du principe de la structure gothique, lequel n'est qu'un système constructif dans lequel les forces sont transmises du haut vers le sol par l'intermédiaire des arcs et des piliers. Ici donc le choix du système des pylônes en $\mathrm{V}$ de Bernard Laffaille prend tout son sens en remplissant à la fois le même rôle d'appui et de reprise des charges. Les vingt-quatre éléments qui définissent la nef sont repensés dans leurs dimensions pour parvenir à la haute nef que l'on peut admirer aujourd'hui. L'arête des pylônes, tournée vers l'intérieur de l'église, est épaissie pour former deux nervures rondes évoquant les colonnettes fasciculées du gothique. Les pylônes sont réunis à leur sommet par une poutre de rive à laquelle est accrochée et suspendue la mince toiture dite en "selle de cheval» car formée d'un paraboloïde hyperbolique autoportant raidi par des tirants. Là aussi, la légèreté et la minceur $(8 \mathrm{~cm})$ de cette " voûte » à double courbure planant à 36 mètres au-dessus du sol de la nef ne sont pas sans évoquer celles des voûtes des cathédrales gothiques. 

parfaitement revendiquées par l'architecte. L'aspect extérieur, scandé par le rythme des éléments verticaux, n'est pas sans rappeler, en négatif, la cathédrale-forteresse SainteCécile d'Albi (Tarn) dont les murs sont formés d'une succession de tourelles demicylindriques. À l'intérieur, le visiteur, placé sur le palier supérieur de l'escalier principal qui descend dans la nef, découvre l'ensemble du volume intérieur et son regard est immédiatement attiré par l'envolée quasi lyrique des nervures des $\mathrm{V}$ et des grandes verrières, nef à propos de laquelle Guillaume Gillet avoua : «Cette nef qui maintenant existe et me dépasse ${ }^{16}$ (fig. 8). François Mauriac a résumé le sentiment général d'intemporalité qui se dégage de Notre-Dame de Royan: «Cette église a beau être de béton, elle nous arrive pourtant du fond des siècles, elle participe de styles auxquels elle n'a rien emprunté $»^{17}$.

Figure 8

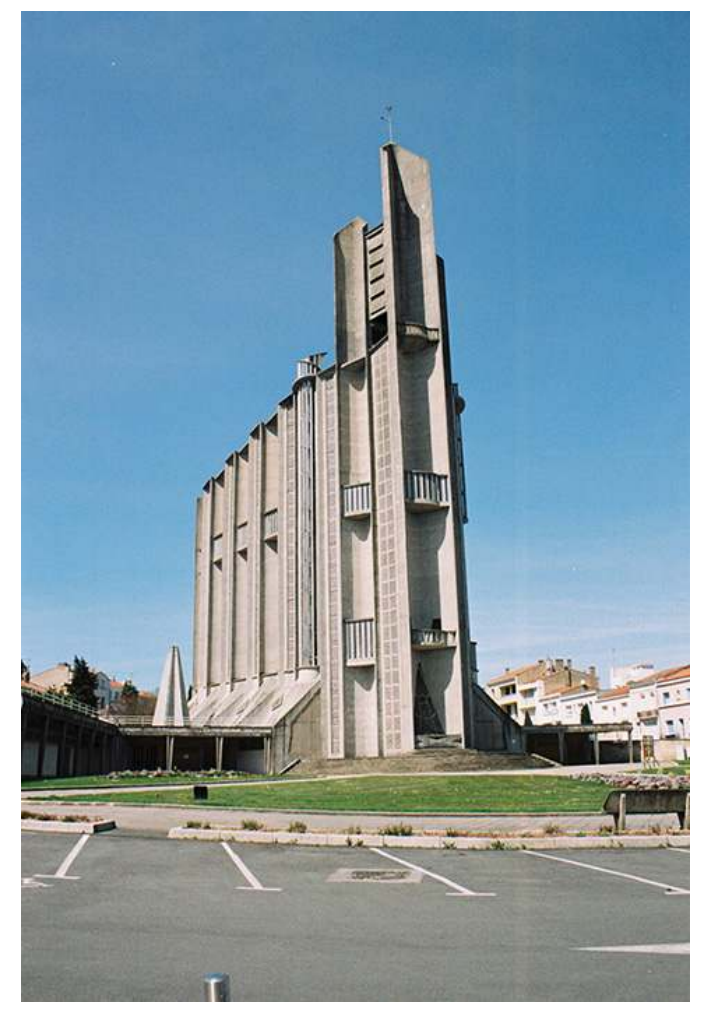

L'église Notre-Dame de Royan, vue d'ensemble depuis le parvis.

Phot. Franck Delorme, 2012. (c) Franck Delorme.

11 En 1968, dix ans après l'inauguration de l'église de Royan, Michel Ragon en faisait encore un des exemples de la «vérité des structures » et établissait le parallèle historique suivant:

«L'architecture gothique, tout comme l'architecture $\mathrm{du} \mathrm{xx}^{\mathrm{e}}$ siècle, est une architecture d'écorché. Toutes les deux tendent à la légèreté et à la gracilité, mais en même temps recherchent la résistance et la puissance. $»^{18}$

Notre-Dame de Royan n'est pas la seule église conçue par Guillaume Gillet. Les prodigieux et étonnants projets qu'il fit pour la basilique de Syracuse (Sicile, 1955-1957) (fig. 9), pour la cathédrale de Liverpool (Royaume-Uni, 1960) et pour l'église Saint-Michel-desGaloubies à Chamalières (Puy-de-Dôme, 1959-1961) - projets qui ont en commun avec 
Royan l'emploi des V Laffaille - ne furent pas suivis de réalisation. En revanche, l'église Saint-Crépin de Soissons (Aisne, 1959-1965) reprend, à une échelle et avec des dimensions plus réduites, les principes formels et constructifs de Royan. La chapelle de la Solitude à Vieux-Condé (Nord, 1961-1966), Saint-Joseph-Travailleur à Avignon (Vaucluse, 1963-1969) et la chapelle du collège du Sacré-Cœur à Châtenay-Malabry (Hauts-de-Seine, 1964-1969) adoptent elles aussi le principe du paraboloïde hyperbolique mais réalisé à l'aide de charpentes en bois lamellé-collé dont les calculs de structure sont dus à un autre ingénieur, Robert Lourdin ${ }^{19}$.

Figure 9

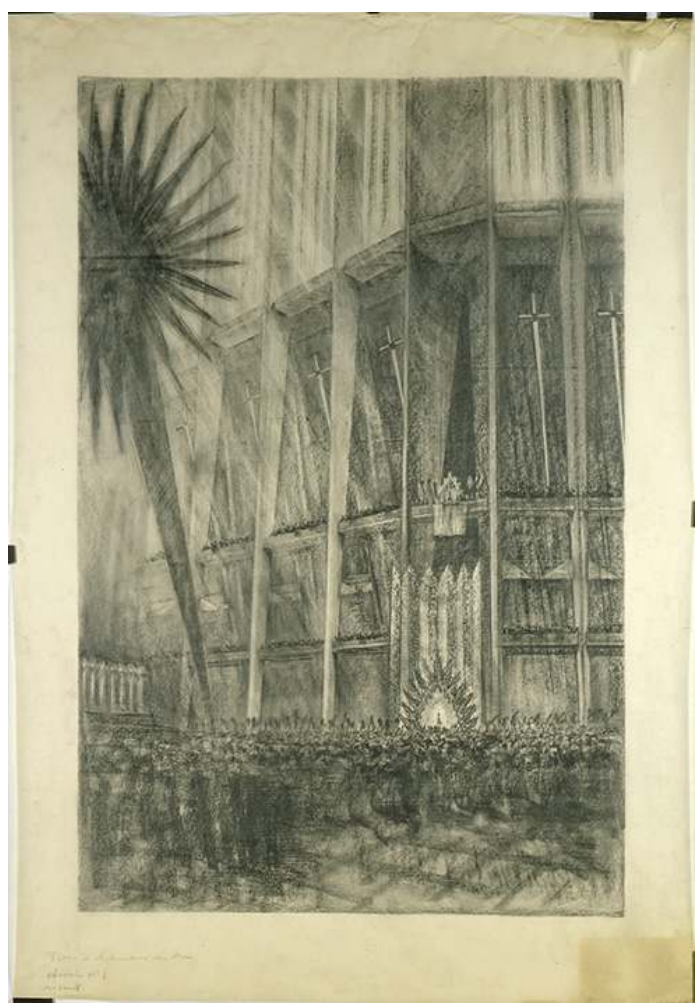

Projet de Guillaume Gillet pour la basilique de Syracuse (Sicile), perspective intérieure de la nef, 1955. SIAF/CAPA, fonds Gillet, 152 IFA 199.

(C) Cité de l'architecture et du patrimoine, Paris.

\section{La liaison des arts à l'épreuve de l'œuvre}

Revenons donc à la question des vitraux et de leur conception. En 1963, pressé par le maire de Royan de s'atteler enfin à la réalisation des vitraux, Guillaume Gillet l'assurait dans une lettre que cette question l'occupait depuis le début du projet : « Le problème des verrières de Notre-Dame de Royan est depuis l'origine de mes études un problème majeur et j'y ai moi-même, depuis mes premières esquisses, pensé, souhaitant grouper, sous ma direction, une équipe d'artistes pour chercher à définir en commun le climat coloré de cette nef $\aleph^{20}$. Heureusement conservé, le premier brouillon de ce courrier apporte, sur les premières intentions de l'architecte, davantage de précisions et de détails, disparus dans la version définitive. Dans ce brouillon, Guillaume Gillet avouait avoir voulu "grouper sous [sa] direction des artistes de son choix », ce qui renseigne sur le "pouvoir » qu'il 
désirait avoir sur les intervenants. Soucieux qu'il fût de faire acte de création importante, il les souhaitait « de qualité et de bonne volonté ». Les propos révèlent l'état d'esprit dans lequel se trouvait l'architecte, alors conscient d'être chargé d'une commande et d'une mission insigne. Il se rêvait peut-être l'égal des bâtisseurs de cathédrales et pensait qu'un édifice de ce type résulte de l'harmonie régnant entre l'architecture et l'art. C'est ce qui semble ressortir des propos finalement retirés : «pour créer dans l'architecture que je dessinais et à laquelle j'osais croire de toutes mes forces un équivalent de ce qui fait notre admiration devant les cathédrales ». Peut-être conscient que ses propos pourraient être pris pour l'expression d'une prétention déplacée, Guillaume Gillet s'en est tenu alors à un discours plus humble. En somme, il s'agit d'une reprise en main d'un projet qui risquait de lui échapper au cours de son achèvement.

Guillaume Gillet se résolut finalement à abandonner l'idée de faire appel à plusieurs artistes, désormais convaincu que le résultat n'aurait sans doute pas un caractère homogène mais risquerait également de ne pas correspondre à l'architecture épurée qu'il avait cherché à produire. Déjà, le doute s'était immiscé quelque temps plus tôt : « Et, plus j'y pense, moins je crois que ce soit des vitraux, des tableaux de verre de couleur qu'il faut faire $»^{21}$. Il renonce donc à appeler des peintres qu'il admire et qui sont pour certains ses amis, car il n'a trouvé parmi eux «que des gens trop occupés par des lithographies, des expositions, des ballets et [...] par des cathédrales de Nevers ${ }^{22}$ pour qu'ils puissent même trouver une journée pour aller voir l'édifice dont il s'agit $»^{23}$. À la lecture des noms Alfred Manessier ${ }^{24}$, Jean Cortot ${ }^{25}$, Raoul Ubac ${ }^{26}$, Antoni Clavé27, André Marchand ${ }^{28}$, Jean Bazaine $^{29}$ - on peut s'interroger sur la capacité de personnalités artistiques si diverses à produire une unité d'ensemble. Alfred Manessier et Raoul Ubac avaient déjà une œuvre marquée par plusieurs ensembles de vitraux. En 1947, le chanoine Lucien Ledeur commandait à Manessier les vitraux de l'église Sainte-Agathe du hameau des Bréseux dans le Doubs. L'architecte Maurice Novarina confiait à Raoul Ubac en 1958 sa première création en matière de vitraux pour l'église d'Ézy-sur-Eure (Eure); en 1961, Ubac collaborait avec Georges Braque pour les vitraux de Varengeville (Seine-Maritime).

Déjà meurtri par l'introduction, à son insu, d'un vitrail financé par souscription et signé Claude Idoux, et ayant repoussé un projet du même artiste (fig. 10), Guillaume Gillet écarte aussi l'idée, avancée par la municipalité, d'organiser un concours entre créateurs. En réalité, celle-ci avait reçu les offres de plusieurs personnes qui souhaitaient proposer des études et des cartons de vitraux. Parmi les diverses propositions figurait celle de la décoratrice et peintre Janie Pichard, introduite par l'entremise de son mari Joseph Pichard, fondateur de la revue L'Art sacré, qui avait suivi avec attention le projet de l'église. Forte de ce soutien, Mme Pichard avait même réussi à faire placer un premier essai de vitrail malgré l'opposition de Guillaume Gillet. Celui-ci retraça rétrospectivement et humoristiquement cet épisode sans toutefois citer le nom de cette « artiste amoureuse de l'église qui s'était mis en tête qu'elle réaliserait les verrières et avait gentiment fait campagne auprès du clergé et des élus pour en poser un échantillon ${ }^{30}$ ». N'ayant pu s'opposer de façon frontale à ce choix, Guillaume Gillet avait dû néanmoins consentir à l'expérience : «L'échantillon, très grand et placé [...] au point le plus haut de l'édifice, sans doute comme un “j'y suis, j'y reste” me fit frémir et je manquai, le voyant en place, de tomber en faiblesse $»^{31}$. 


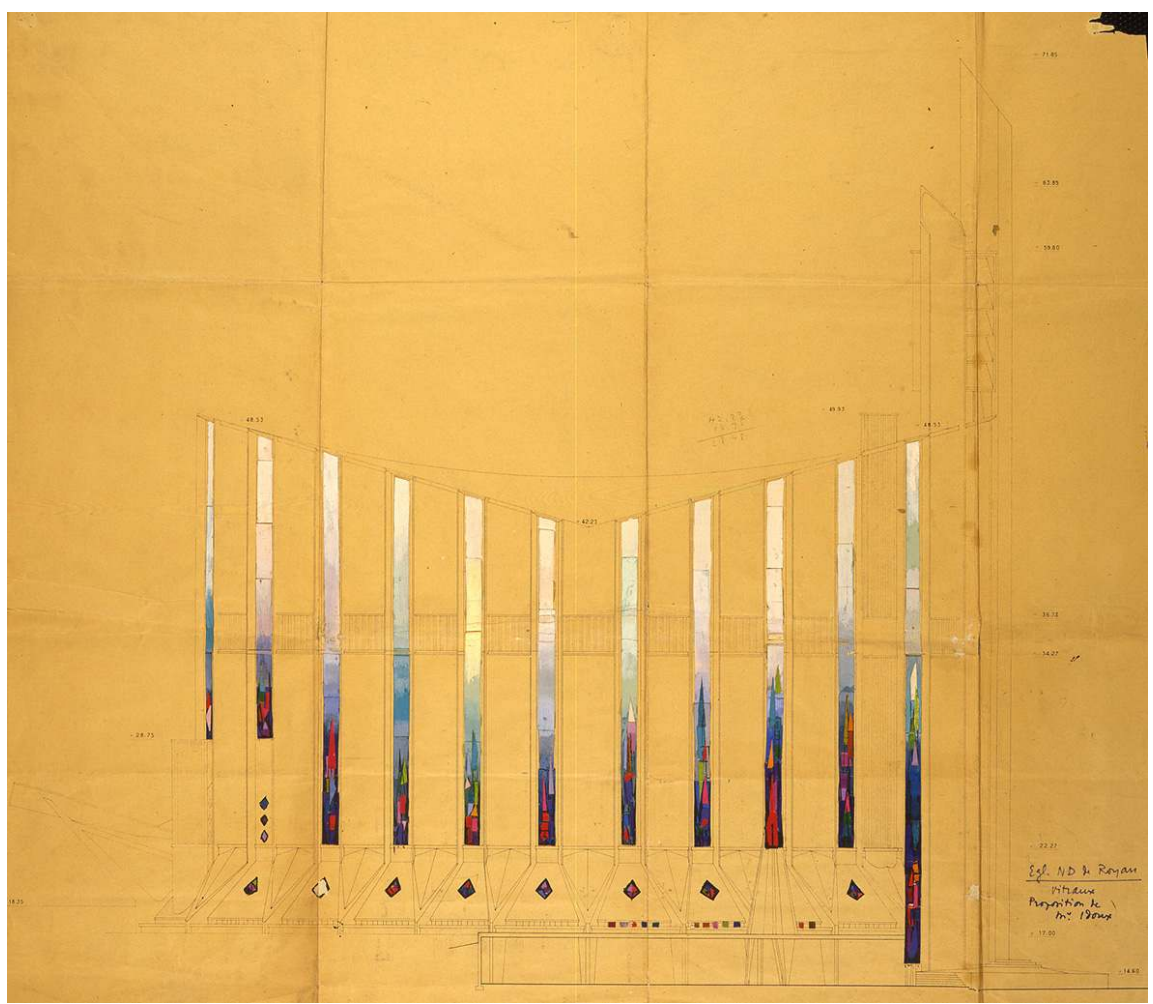

Projet du peintre Claude Idoux pour les verrières de l'église Notre-Dame de Royan, non daté. SIAF/ CAPA, fonds Gillet, 152 IFA 514.

(C) Cité de l'architecture et du patrimoine, Paris.

\section{L'unité sous la conduite d'un chef unique}

En fait, sous la notion de liaison des arts, Guillaume Gillet cherchait à comprendre pourquoi dans l'architecture de son époque les artistes contemporains ne trouvaient pas place comme par le passé. Selon ses propres mots,

«le seul fait que la question soit posée et le terme de liaison employé semble prouver qu'il existe dans les esprits d'aujourd'hui une notion a priori de séparation de ces arts qui dans toute l'histoire connue jusqu'à nous ne nous ont laissé que les témoignages et les témoins de leur union intime, de leur mariage essentiel, originel, évident, nécessaire ${ }^{32}$.»

17 À l'appui de cette constatation et l'esprit sans doute occupé par les vitraux de Royan, il rappelait qu'aux temps anciens, "aucun d'entre eux n'a dissocié l'architecture des arts plastiques : le peintre et le sculpteur et l'architecte ont fait équipe évidemment aussi bien à Ajanta ${ }^{33}$ qu'à Saint-Savin ${ }^{34}$, qu'à Chartres sur le programme d'un Clergé ${ }^{35}$ ».

Guillaume Gillet avait tout de même conscience qu'une œuvre collective, pour être menée à terme et aboutir à cette union, avait besoin, la plupart du temps, d'un maître d'œuvre qui fixe le programme et l'objectif, contrôle l'apport de chacun par rapport à un projet esthétique. Et de citer «Versailles où Le Brun, le peintre a été le chef d'orchestre d'un concert où les premiers violons étaient Le Nôtre et Mansart, et dont le compositeur était le Roi en personne ». Il reprend donc à son compte la fameuse expression de "chef d'orchestre » mais il est bien évident que, dans son esprit, ce chef ne saurait être que 
l'architecte lui-même. Le sens de liaison entre les arts, dans l'esprit de Guillaume Gillet, n'est donc pas synonyme de responsabilité collective et d'égalité entre les intervenants. Confronté concrètement au problème, Guillaume Gillet pressent qu'il appartient à l'architecte de maîtriser l'apport de chacun des artistes dans le respect de l'œuvre d'ensemble - et non pas de l'œuvre collective -, et de parvenir à l'unité souhaitée. À l'intérieur de l'église de Royan, des œuvres d'artistes - essentiellement des sculpteurs sont introduites de manière parcimonieuse sans prendre une place visuelle importante dans l'espace. Par exemple, une Vierge sculptée par Gaston Watkin ${ }^{36}$ (1916-2011) reçoit l'assentiment de Guillaume Gillet, puisque celui-ci est à l'origine du choix de l'artiste et de la commande. Afin d'accorder les différents détails avec l'espace, l'architecte dessine le mobilier (fig. 11) comme il le fait pour les autres églises dont il est l'auteur.

Figure 11

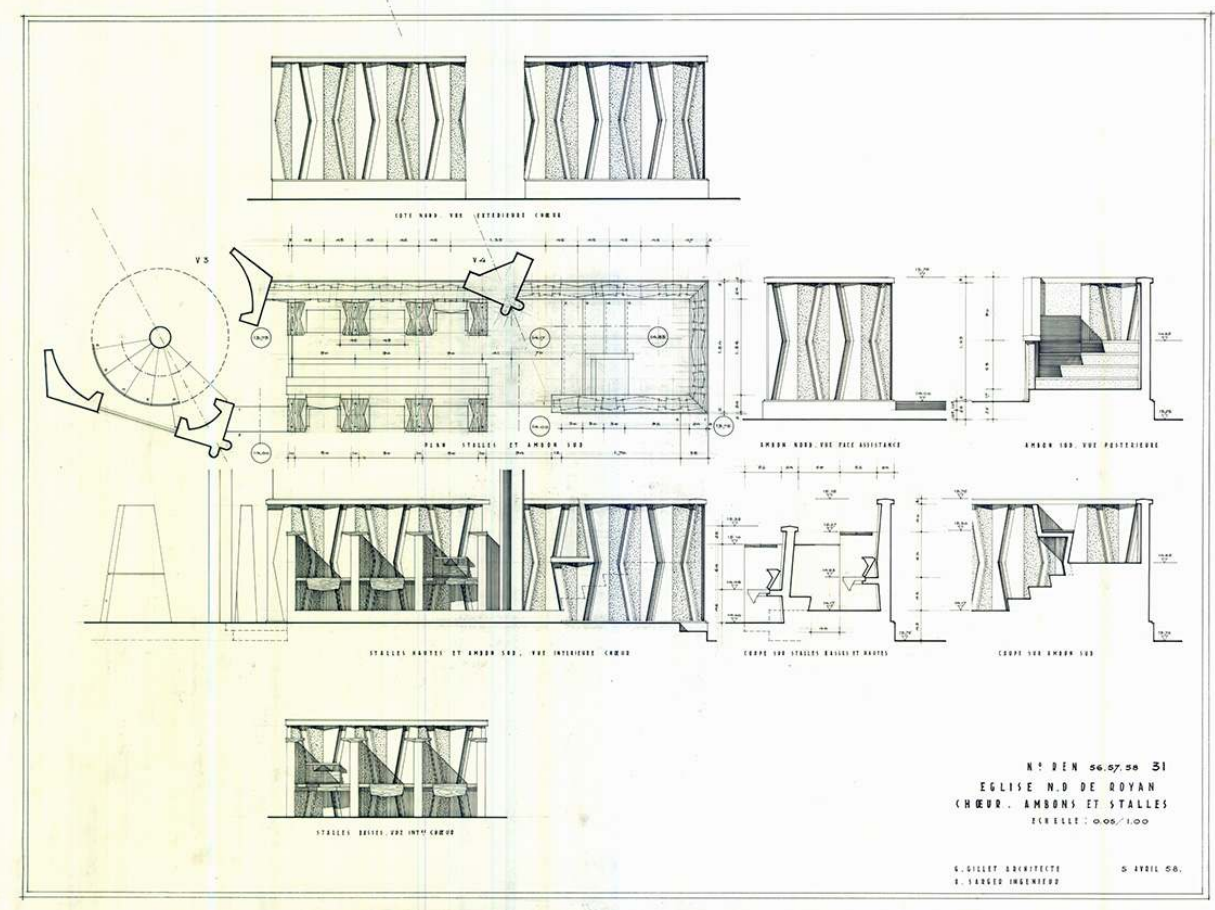

Projet de Guillaume Gillet pour les ambons et les stalles du chœur de l'église Notre-Dame de Royan, 5 avril 1958. SIAF/CAPA, fonds Gillet, 152 IFA 643/5.

(c) Cité de l'architecture et du patrimoine, Paris.

L'intervention « artistique » la plus importante reste donc le programme de verrières qui se déploient sur l'ensemble des baies. La surface et la présence spatiale des futurs vitraux obligeaient, comme on l'a vu, l'architecte à être prudent dans le choix de son concepteur et exécuteur. Dans le texte de ses mémoires, Gillet a donné lui-même la clé pour comprendre comment s'opéra ce choix. "Ce fut en fin de compte le très modeste et simple Henri Martin-Granel qui mit au point la solution conforme à mes désirs d'un appareillage de briques de verre, une grisaille colorée qui se marie discrètement à l'architecture $\Perp^{37}$. Né en 1914, Henri Martin-Granel suivit d'abord des études d'architecture à l'École des beaux-arts de Paris dans l'atelier de Charles Lemaresquier. Passionné d'archéologie, il pratique également la sculpture et ses premières œuvres sont un gisant de saint Louis à Bizerte (Tunisie, 1954) pour commémorer la mort du roi à 
Carthage pendant la huitième croisade, et le mémorial de l'anti-esclavagiste Louis Delgrès à Pointe-à-Pitre en Guadeloupe (1956). L'architecte Jean Le Couteur, rencontré en 1934 aux Beaux-Arts, lui demande d'être son collaborateur sur le chantier de l'église NotreDame à Bizerte en Tunisie. Artiste profondément croyant, ce fut pour cet édifice qu'il réalisa ses premiers vitraux. Henri Martin-Granel était plutôt tenté par le figuratif, comme en témoignent les vitraux qu'il conçut pour la cathédrale d'Alger à la demande des architectes Paul Herbé et Jean Le Couteur en 1963 (fig. 12), prédilection qu'il conserva tout au long de sa carrière. Martin-Granel est avant tout « un créateur original dans l'univers du vitrail ${ }^{38}$ » comme le qualifie Véronique David, historienne de l'art spécialiste du vitrail contemporain. Il inventa plusieurs techniques de vitrail dont le «sandwich de verre et de briques » selon la propre expression de l'artiste lui-même, emprisonnant des dalles de verre entre deux "tranches" minces de briques perforées, formant ainsi des sortes de claustras constituant de véritables «murs de verre " comme au Studium des Dominicains à Toulouse (Haute-Garonne, 1960). C'est cette technique de vitrail qui fut d'abord envisagée pour les verrières de Notre-Dame à Royan avant que le peintre verrier n'en propose une autre de son invention à l'architecte.

Figure 12

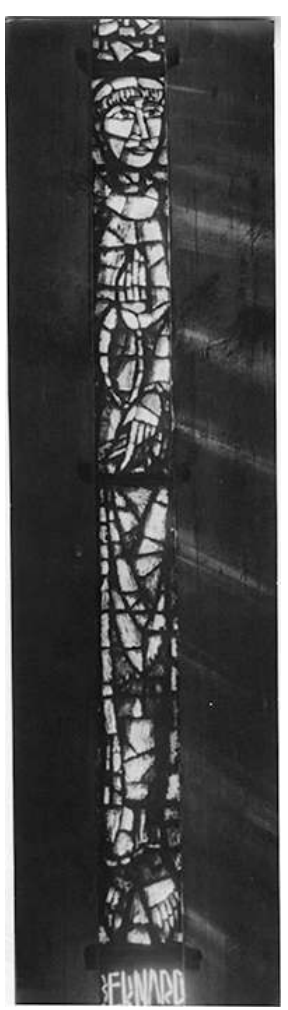

Vue d'un vitrail d'Henri Martin-Granel pour l'église Notre-Dame de France à Bizerte (Tunisie) par Jean Le Couteur et Paul Herbé, non daté. SIAF/CAPA, fonds Gillet, 152 IFA 1033/2.

(c) Cité de l'architecture et du patrimoine, Paris

21 Henri Martin-Granel a apporté à Guillaume Gillet un procédé technique de vitrail - dérivé de la dalle de verre - définissant un principe esthétique qu'il avait déjà expérimenté pour les petits vitraux de la couverture des fonts baptismaux en 1956-1957 (fig. 13) et qu'il utilisera également à l'église Saint-Crépin de Soissons. 
Figure 13

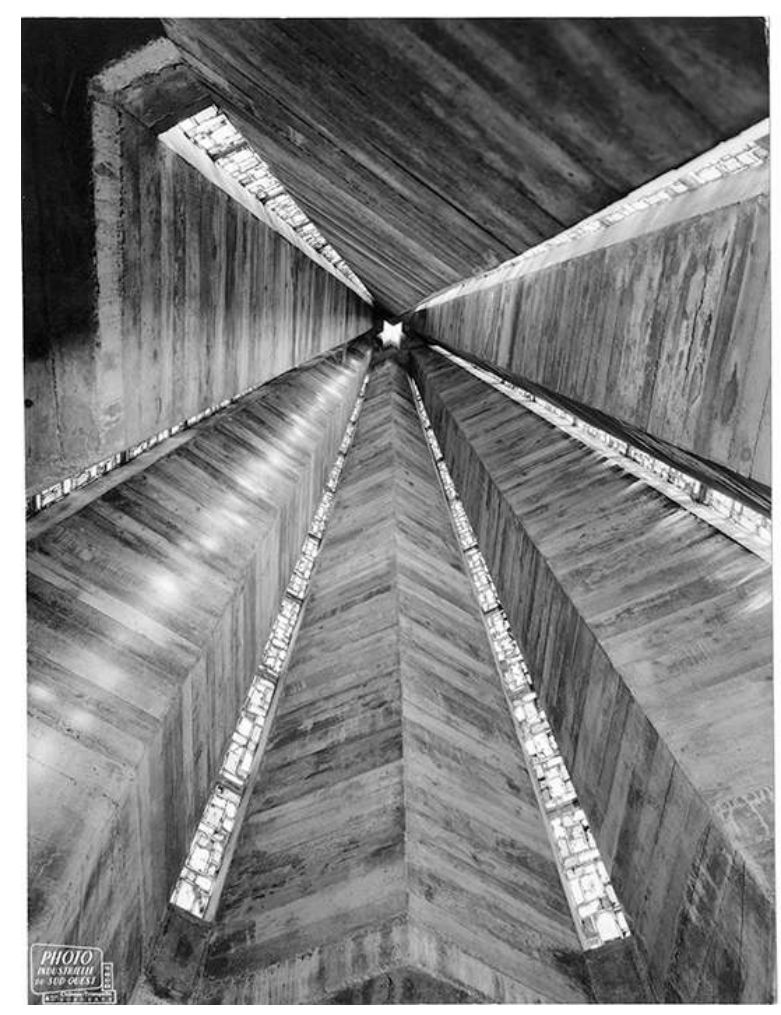

Vue en contreplongée de la couverture des fonts baptismaux de Notre-Dame de Royan avec les vitraux d'Henri Martin-Granel, 12 janvier 1959. SIAF/CAPA, fonds Gillet, 152 IFA 1033/2.

Phot. Photo industrielle du Sud-Ouest. (C) Cité de l'architecture et du patrimoine, Paris.

Comme il l'expliquait dans une note, Henri Martin-Granel eut en effet l'idée d'enchâsser dans les joints de ciment de la dalle de verre des lames de verre cathédrale blanc posées sur chant et divisant la verrière en alvéoles. Il démontrait à l'architecte que

«l'intérêt de ces alvéoles est multiple: du point de vue plastique, par la vision latérale et d'en dessous (important dans le cas de verrières de grande hauteur) cette résille crée des transparences, des demi-teintes, des irisations, qui augmentent la légèreté et le moelleux, en même temps que la lumière est diffusée; le dessin géométrique de la résille, matérialisé par la tranche du verre éclairée, se profile de $6 \mathrm{~cm}$ en avant du vitrail et [est] décalé par la perspective ; tout cet ensemble donne au vitrail une profondeur, une troisième dimension, en même temps qu'une grande légèreté et luminosité ${ }^{39}$ » (fig. 14). 
Figure 14

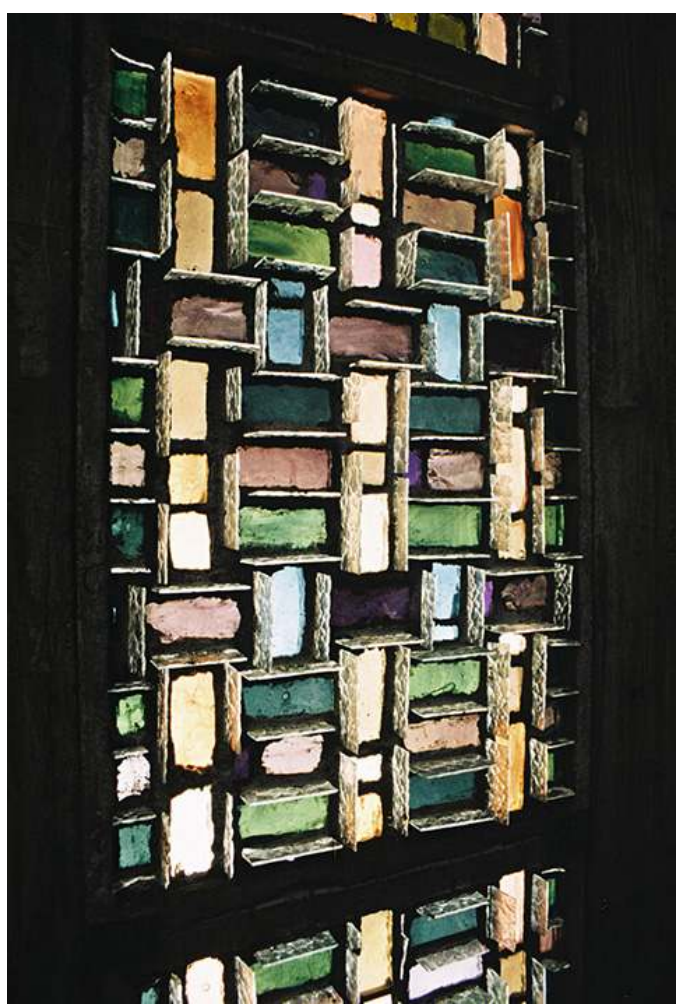

Détail d'un vitrail d'une verrière de la nef de Notre-Dame de Royan, réalisé en dalle de verre sur un motif en damier par Henri Martin-Granel, avec les lames de verre blanc.

Phot. Franck Delorme, 2012. ( ) Franck Delorme.

Si le peintre verrier apporte donc la technique de mise en œuvre du vitrail, c'est visiblement bien l'architecte qui oriente le parti esthétique vers une abstraction géométrique sur une variation de motifs en chevrons, en losanges, etc. L'expression de « mise au point » employée par Guillaume Gillet ainsi que les esquisses de sa main laissent peu de doute sur le manque de liberté de création auquel a été contraint, en définitive, le peintre verrier. Sans être péjoratifs, les qualificatifs «modeste » et «simple » employés par Guillaume Gillet pour définir Henri Martin-Granel disent néanmoins combien ce dernier dut réduire son rôle à l'exécution d'un principe arrêté avec l'architecte. Pressé par la nécessité d'exécuter rapidement l'ensemble des verrières, le peintre verrier a travaillé sans produire de cartons préalables, en choisissant une harmonie colorée et en appliquant cinq motifs géométriques (chevrons, diagonales, escaliers, triangles, damiers) (fig. 15) qu'il distribue symétriquement sur les deux côtés de la nef. Ce mode de mise en œuvre a d'ailleurs été source de mécontentement de la part de Guillaume Gillet, notamment pour les « deux verrières du chœur de l'église qui ne rendent pas du tout » ce qu'il en attendait. Il juge que « leur dessin en chevrons n'accompagne pas bien le geste vertical $»^{40}$ (fig. 16). 
Figure 15

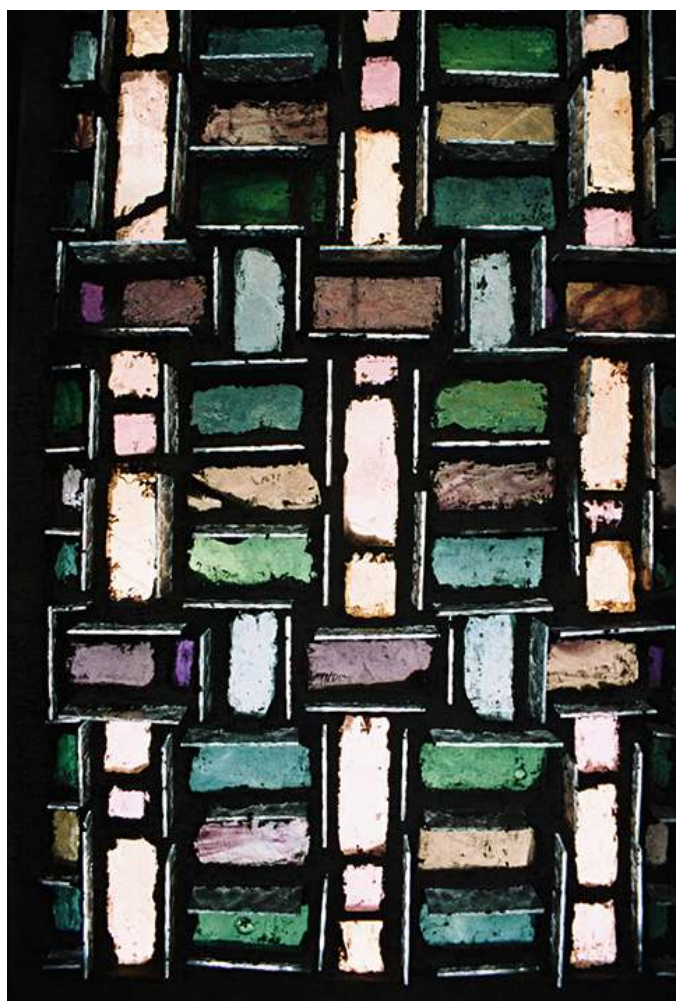

Détail d'un vitrail d'une verrière de la nef de Notre-Dame de Royan, réalisé en dalle de verre sur un motif en damier par Henri Martin-Granel.

Phot. Franck Delorme, 2012. ( ) Franck Delorme. 
Figure 16

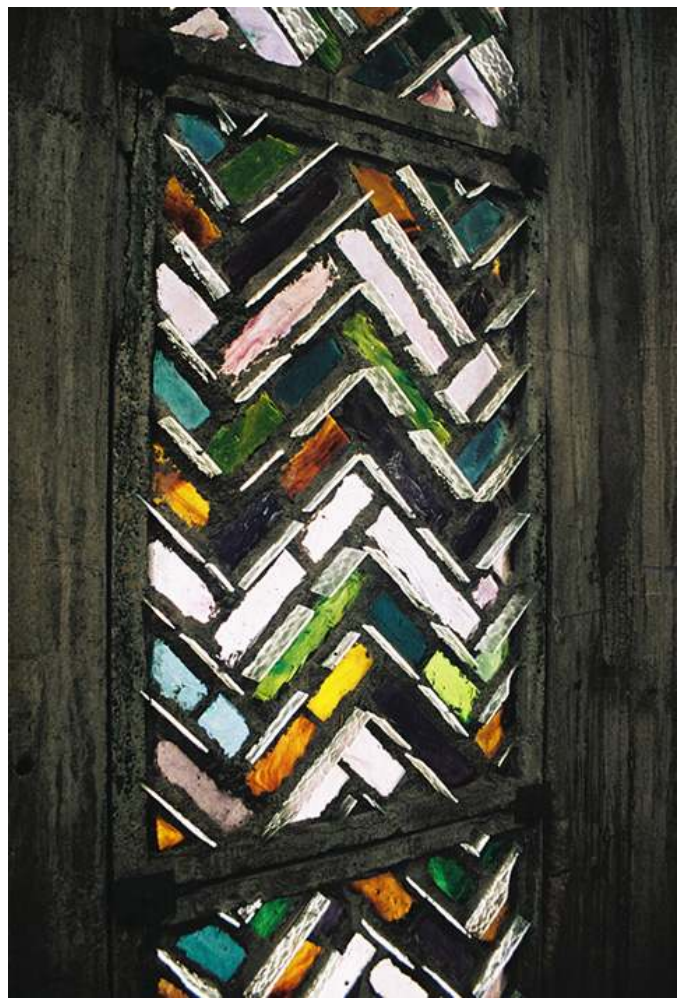

Détail d'un vitrail d'une verrière de la nef de Notre-Dame de Royan, réalisé en dalle de verre sur un motif en chevrons par Henri Martin-Granel.

Phot. Franck Delorme, 2012. ( ) Franck Delorme.

Il précise que, pour ces deux verrières, il lui avait « demandé de reprendre le joli dessin en escaliers des deux verrières derrière l'orgue [...] qui leur répondent en plan ». En effet, il avait explicitement précisé qu'il lui semblait « évident que c'est la maille en escalier [...] qu'il faut reprendre - probablement avec une dominante de bleus à laquelle arrive insensiblement la gradation des verrières déjà en place et pour tenir autant que possible le coup auprès du vitrail triangulaire ${ }^{41}$ [ndlr : celui du chœur par Claude Idoux] » (fig. 17). 
Figure 17

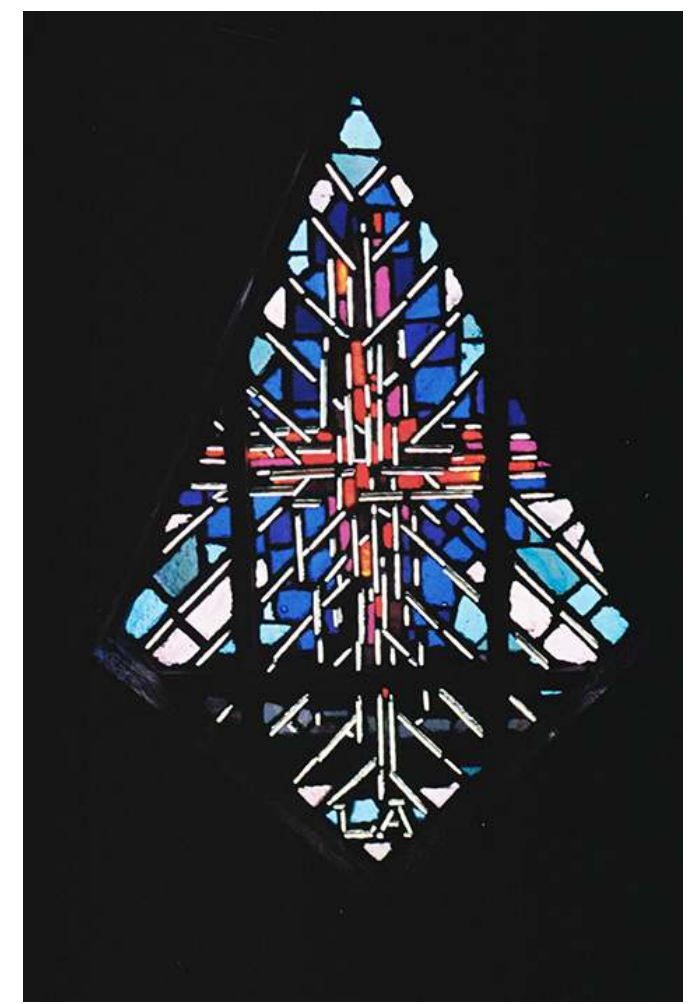

Vitrail en losange de la galerie inférieure de l'église Notre-Dame de Royan, réalisé en dalle de verre par Henri Martin-Granel.

Phot. Franck Delorme, 2012. ( ) Franck Delorme.

$\mathrm{Au}$ moment où était conçu le programme des vitraux de Royan, Guillaume Gillet construisait l'église Saint-Crépin à Soissons - la première pierre en fut posée en 1962 et le gros œuvre achevé en 1964. L'église de Soissons est, selon son architecte, l'occasion «d'appliquer les principes monumentaux de Royan à une échelle moindre et [...] de retrouver entre la petite et la grande église l'air de famille que nos ancêtres ont su si bien conserver entre l'église de village et la cathédrale $»^{42}$. Entre les deux édifices, il est indéniable et incontestable qu'il existe une ressemblance étroite et évidente: même silhouette oblongue, même inflexion du profil, même élan vertical, même ponctuation du clocher, etc. La similitude tient bien évidemment en grande partie à l'emploi des mêmes éléments en béton armé. La couverture de Soissons ressemble par son profil à celle de Royan, mais par mesure d'économie elle fut réalisée à l'aide d'un système d'arcs en bois lamellé-collé tel que l'architecte commençait à le développer au même moment ${ }^{43}$. Réalisés de 1964 à 1965, les vitraux de Soissons prennent place entre les mêmes pylônes en V qu'à Royan. Le programme coloré exploite une progressivité des tonalités qui vont d'une gamme de bleu, de jaune, de vert et de rose dans la nef à une dominante rouge très soutenue dans le chœur. Les motifs géométriques sont les mêmes que ceux utilisés à Royan suivant la même implantation symétrique. 


\section{Conclusion}

L'ensemble décoratif de l'église de Novarina à Assy est le résultat du travail d'un collectif d'artistes intervenant sous la direction d'un architecte (et d'autorités religieuses) qui laisse chacun libre de son expression personnelle. Notre-Dame de Royan est au contraire tout entière soumise à la volonté d'un architecte qui oriente et définit l'unité artistique et architecturale. D'une liaison souhaitée entre l'architecture et les arts plastiques destinés à en prolonger l'esprit et l'atmosphère (fig. 18), Guillaume Gillet a abouti à une union dont il est le principal maitre d'œuvre et dans laquelle il semble bien que l'architecte et l'artiste n'aient pas la même implication ni la même liberté d'expression. L'expression du peintre verrier est soumise à la volonté de l'architecte qui poursuit une idée esthétique. André Wogenscky disait, à propos du recours qu'il faisait des artistes dans ses édifices, vouloir «appeler le sculpteur, parfois le peintre, pour qu'il continue à renforcer l'intensité [qu'il a] essayé de donner par l'architecture ${ }^{44}$ ». Il apparaitt bien que les vitraux d'Henri Martin-Granel à Notre-Dame de Royan parachèvent et renforcent l'intensité que Guillaume Gillet a souhaité atteindre dans la conception d'un nouvel espace sacré en soumettant pour cela l'expression de l'artiste à la volonté de l'architecte.

Figure 18

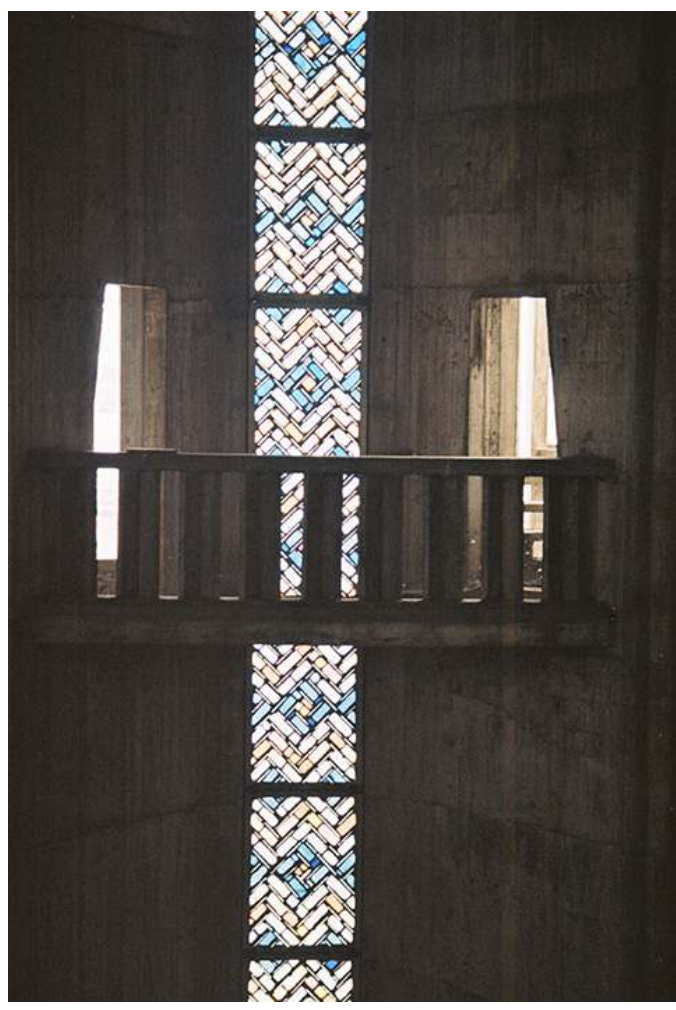

Une des verrières de la nef de Notre-Dame de Royan close par des panneaux de vitraux en dalle de verre par Henri Martin-Granel sur un motif en double chevrons.

Phot. Franck Delorme, 2012. (c) Franck Delorme. 


\section{NOTES}

1. - Sur la reconstruction de la ville de Royan : RAGOT, Gilles (dir.). L'invention d'une ville. Royan 50. Paris : Éditions du patrimoine, coll. "Cahiers du patrimoine », 2003 ; également RAGOT, Gilles. "L'invention du balnéaire "cinquante » à Royan », In Situ [En ligne], 4|2004, mis en ligne le 19 avril 2012, consulté le 17 mai 2017. URL: http://insitu.revues.org/2260 ; DOI : 10.4000/ insitu. 2260.

2. - L'église Notre-Dame de Royan a été classée monument historique en 1988, un peu plus d'un an après le décès de son architecte.

3. - Notre-Dame de Royan fut rapidement élevée au rang d'icône de la modernité, bien avant son achèvement complet. La maquette fut présentée en février 1959 au musée d'Art moderne de New York dans une exposition sur l'architecture religieuse contemporaine. Le projet a également figuré en bonne place au XI ${ }^{\mathrm{e}}$ Salon d'art sacré (1961) à Paris.

4. - Ce n'est pas l'objet ici de retracer le projet de reconstruction de l'église de Royan, nous renvoyons le lecteur à l'ouvrage : DELORME, Franck. L'église Notre-Dame de Royan, Guillaume Gillet et le gothique moderne. Bordeaux : Le Festin, 2012.

5. - L'ingénieur Bernard Laffaille, diplômé de l'École centrale des arts et manufactures, développe dès les années 1930 les propriétés constructives des surfaces gauches en coque de béton armé, réalisant les premiers conoïdes (1927) puis les premiers paraboloïdes hyperboliques (1933) qu'il utilisera pour réaliser la couverture de l'église de Royan. Parallèlement, il engage ses réflexions vers la préfabrication lourde. Dès 1934, il conçoit le «V Laffaille " qu'il mettra largement en œuvre après-guerre dans les rotondes de la SNCF entre 1944 et 1950, et à grande échelle à l'église de Royan dont son élève René Sarger terminera les études de la structure avec Guillaume Gillet.

6. - Les archives de Guillaume Gillet ont été données à l'État et sont déposées au Centre d'archives d'architecture du $\mathrm{xx}^{\mathrm{e}}$ siècle de la Cité de l'architecture et du patrimoine, où elles sont consultables. L'instrument de recherche est accessible par la base de données Archiwebture à l'adresse : http://archiwebture.citechaillot.fr/fonds/FRAPN02_GILGU [consulté le 17/05/2017].

7. - Nous avons pu consulter en 2011 les archives du peintre verrier Henri Martin-Granel en possession de sa famille.

8. - GILLET, Guillaume. "La liaison de l'art monumental et des arts plastiques ». Communication faite devant la commission de l'Équipement culturel du Ve Plan, tapuscrit, 2 avril 1965 (SIAF/ CAPA, fonds Gillet, cote 152 IFA 2562/1).

9. - Ibid.

10. - Lettre de Guillaume Gillet à l'amiral Meyer, maire de Royan, 6 novembre 1963 (SIAF/CAPA, fonds Gillet, cote 152 IFA 2484/1).

11. - GILLET, Guillaume. «Je suis architecte ». Manuscrit, vers 1962 (SIAF/CAPA, fonds Gillet, cote 152 IFA 2538/2).

12. - MONNIER, Gérard. L'architecture moderne en France. Une histoire critique. 1918-1950. Paris : éditions Philippe Sers, 1990, p. 68.

13. - GILLET, Guillaume. Op.cit.

14. - Ibid.

15. - DELORME, Franck. Op. cit. Voir aussi : Id. « Guillaume Gillet et le gothique moderne ». Le Point riche, bulletin des Amis de Louis Mazetier, $\mathrm{n}^{\circ}$ 8, juin 2010, p. 21-43.

16. - GILLET, Guillaume. «Je suis architecte », manuscrit cité.

17. - MAURIAC, François. Nouveau bloc-notes. Paris : Flammarion, 1961, p. 374.

18. - RAGON, Michel. Article sans titre. Les Annales Conferencia, mars 1968. 
19. - Robert Lourdin collabora également avec Guillaume Gillet pour la couverture de la salle des pas perdus de l'École nationale de la magistrature à Bordeaux (Gironde), autre réalisation majeure de l'architecte.

20. - Lettre de Guillaume Gillet à l'amiral Meyer, maire de Royan, 26 novembre 1963 (SIAF/CAPA, fonds Gillet, cote 152 IFA 2484/1).

21. - Ibid.

22. - La cathédrale Saint-Cyr et Sainte-Julitte de Nevers (Nièvre) est un des premiers grands édifices anciens pour lequel fut engagé après 1945 un programme de vitraux contemporains. Initié dans les années 1950 avec les vitraux de Raoul Ubac pour le chœur roman, le programme se poursuivit pendant un demi-siècle, jusqu'aux dernières verrières de Jean-Michel Alberola, posées en 2009.

23. - Note de Guillaume Gillet relative aux verrières à exécuter, 8 janvier 1964 (SIAF/CAPA, fonds Gillet, cote 152 IFA 2491/4).

24. - Alfred Manessier (1911-1993), d'abord attiré par l'architecture, finit par choisir la peinture au cours de sa scolarité à l'École des beaux-arts de Paris ; il est un des maîtres de la nouvelle École de Paris. Manessier créa ses premiers vitraux au début des années 1930 (chapelle du Pouldu, Clohars-Carnoët, Finistère). Après la guerre, il reçut d'autres commandes de vitraux, notamment ceux de l'église Sainte-Agathe des Bréseux (Doubs) qui restent une des créations marquantes de l'union de l'art sacré et de l'art abstrait.

25. - Jean Cortot (né en 1925), peintre élève d'Othon Friesz, illustrateur de nombreux ouvrages.

26. - Raoul Ubac (1910-1985), proche des surréalistes, réalise ses premiers vitraux et mosaïques dans les années 1950, et collabore avec Georges Braque à Varengeville (Seine-Maritime).

27. - Antoni Clavé (1913-2005), peintre catalan, d'abord dessinateur, décorateur de théâtre et concepteur d'affiches de cinéma. Sa rencontre de Picasso, en 1944, est décisive : comme le maître, Clavé est passionné de corridas, un des thèmes des nombreuses lithographies dont il est l'auteur.

28. - André Marchand (1907-1997) se forme en fréquentant assidument le musée du Louvre ; il est un des fondateurs en 1945 du Salon de mai avec Manessier. Ses thèmes de prédilection sont la Provence, la Camargue et la Méditerranée.

29. - Jean Bazaine (1904-2001), figure majeure de la nouvelle École de Paris et de la peinture d'avant-garde, consacra une grande partie de son activité à la création de vitraux pour des églises nouvelles (Assy, Audincourt) et anciennes (Saint-Séverin, Saint-Dié).

30. - GILLET, Guillaume. Texte intitulé « Vitraux ». Dans « Je suis architecte », manuscrit cité.

31. - Ibid.

32. - Id. « La liaison de l'art monumental et des arts plastiques ». Communication faite devant la commission de l'Équipement culturel du Ve Plan. Document cité.

33. - Ajantâ est un monastère bouddhiste situé dans l'État indien du Maharashtra, constitué de 30 grottes artificielles dont l'intérieur est orné de peintures et de sculptures. L'ensemble a été classé au patrimoine mondial par l'Unesco en 1983.

34. - Saint-Savin-sur-Gartempe (Vienne) est célèbre pour son église abbatiale dont la voûte de la nef est recouverte de peintures murales des $\mathrm{XII}^{\mathrm{e}}$ et XIII ${ }^{\mathrm{e}}$ siècles ; une copie de cette voûte, réalisée de 1940 à 1943, est visible au musée des Monuments français à la Cité de l'architecture et du patrimoine à Paris.

35. - GILLET, Guillaume. « La liaison de l'art monumental et des arts plastiques ».

36. - Gaston Watkin, élève de Paul Landowski à l'École des beaux-arts de Paris, obtint en 1946 le Grand Prix de Rome de sculpture pour son œuvre Saint Étienne lapidé. Il eut toute sa carrière durant de nombreuses et prestigieuses commandes publiques pour des bâtiments civils ou religieux. Guillaume Gillet fit appel à lui pour plusieurs chantiers : Ombrelles fluides au collège Jules-Régnier à Brienne-le-Château (Aube) en 1968, mur-décor au collège de Montrouge (Hautsde-Seine) en 1975 et mur-décor au collège de la Triloterie à Royan en 1976. Il était aussi à l'aise 
avec la pierre que le métal. Il réalisa des statues mais aussi des vitraux, notamment un cycle sur la vie de saint Pierre à Aurec-sur-Loire (Haute-Loire).

37. - GILLET, Guillaume. Texte intitulé « Vitraux », cité supra.

38. - DAVID, Véronique. "Martin Granel (1914-2008), un créateur original dans l'univers du vitrail ». Le Point riche (bulletin de l'association des Amis de Louis Mazetier), $\mathrm{n}^{\circ}$ 10, juin 2012, p. 25-37.

39. - Henri Martin-Granel, dans une note à Guillaume Gillet au sujet des vitraux de Notre-Dame de Royan (SIAF/CAPA, fonds Gillet, cote 152 IFA 2491/4).

40. - Lettre de Guillaume Gillet à Henri Martin-Granel, 20 août 1970 (SIAF/CAPA, fonds Gillet, cote 152 IFA 2491/4).

41. - Lettre de Guillaume Gillet à Henri Martin-Granel, 17 avril 1967 (SIAF/CAPA, fonds Gillet, cote 152 IFA 2491/4).

42. - Lettre de Guillaume Gillet à Mgr Collangette évêque de Soissons, $1^{\text {er }}$ octobre 1958 (SIAF/ CAPA, fonds Gillet, cote 152 IFA 2200/1).

43. - Sur l'église et les vitraux de Soissons, voir: DAVID, Véronique et DELORME, Franck. "L'église Saint-Crépin et Saint-Crépinien à Soissons et ses vitraux (1959-1965) ». Le Point riche (bulletin des Amis de Louis Mazetier), n 10, 2012, p. 19-24.

44. - André Wogenscky, interrogé en 1999 sur ses rapports avec les arts et notamment la sculpture (interview inédite réalisée par l'École nationale supérieure d'architecture de Grenoble et non diffusée).

\section{RÉSUMÉS}

Par sa haute silhouette, l'église Notre-Dame de Royan domine la ville et symbolise sa renaissance. L'édifice est une des manifestations majeures du renouveau de l'architecture religieuse après 1945. Son architecte Guillaume Gillet, avec l'ingénieur Bernard Laffaille, y a mis en œuvre un langage gothique moderne qui renoue avec la règle liant principe constructif et expression architecturale. Dans ce squelette de béton armé s'insère un programme de vitraux en dalles de verre réalisé par le peintre verrier Henri Martin-Granel. De l'architecte et de l'artiste, se pose la question de la paternité de l'œuvre plastique ; appartient-elle à l'un ou à l'autre ? Est-elle le fruit d'une collaboration des deux créateurs ou bien de la subordination du second à la volonté esthétique du premier? Notre-Dame de Royan et ses vitraux sont un support pour tenter de saisir le sens de la liaison de l'art monumental et des arts plastiques telle que Guillaume Gillet l'exposait en 1965, au moment où il essayait de définir le parti esthétique des vitraux de NotreDame.

With its soaring silhouette, the Notre-Dame church at Royan dominates the city and symbolises its rebirth after the Second World War. The building is one of the major manifestations of the revival of religious architecture after 1945. Its architect, Guillaume Gillet, with the engineer Bernard Lafaille, invented a Modern Gothic idiom that gave new life to the principle of uniting constructive and architectural expression. The structure in reinforced concrete comprises a programme of stained glass designed by the glass painter Henri Martin-Granel. Between the architect and the artist, the question of the authorship of the plastic work can be posed: does it belong to the one or to the other? Is the final work the result of a collaboration between two designers or the subordination of the glass painter to the architect's aesthetic will? The church 
and its stained-glass windows offer a way to understand the meaning of the connection between monumental art and visual arts. Guillaume Gillet addressed this issue in 1965, when he was trying to define the aesthetic options for the stained glass of Notre-Dame in Royan.

\section{INDEX}

Mots-clés : Royan, Charente-Maritime, Guillaume Gillet, Claude Ferret, Louis Simon, Maurice Novarina, Paul Herbé, Jean Le Couteur, André Wogenscky, Bernard Laffaille, Robert Lourdin, Henri Martin-Granel, Claude Idoux, Alfred Manessier, Georges Braque, Fernand Léger, Raoul Ubac, Georges Rouault, Jean Bazaine, Jean Cortot, Antoni Clavé, André Marchand, Gaston Watkin, architecture religieuse, art sacré, église, vitraux, Trente Glorieuses, langage gothique moderne, dalle de verre, liaison de l'art monumental et des arts plastiques, basilique de Syracuse, cathédrale de Liverpool, Saint-Michel des Galoubies, Saint-Crépin de Soissons, chapelle de la Solitude de Vieux-Condé, Saint-Joseph-Travailleur d'Avignon, collège du Sacré-Cœur de Châtenay-Malabry

Keywords : Royan, Charente-Maritime, Guillaume Gillet, Claude Ferret, Louis Simon, Maurice Novarina, Paul Herbé, Jean Le Couteur, André Wogenscky, Bernard Laffaille, Robert Lourdin, Henri Martin-Granel, Claude Idoux, Alfred Manessier, Georges Braque, Fernand Léger, Raoul Ubac, Georges Rouault, Jean Bazaine, Jean Cortot, Antoni Clavé, André Marchand, Gaston Watkin, religious architecture, sacred art, church, stained glass, Trente Glorieuses, Modern Gothic language, glass slab, llink between monument art and visual arts, Syracuse basilica, Liverpool cathedral, Saint-Michel des Galoubies, Saint-Crépin de Soissons, chapel of the Solitude de VieuxCondé, Saint-Joseph-Travailleur (Avignon), collège du Sacré-Cœur (Châtenay-Malabry)

\section{AUTEUR}

\section{FRANCK DELORME}

Historien de l'architecture, attaché de conservation, Cité de l'architecture et du patrimoine fdelorme@citechaillot.fr 